Research Paper

\title{
Epithelioid Trophoblastic Tumors: Treatments, Outcomes, and Potential Therapeutic Targets
}

\author{
Junjun Yang ${ }^{1 *}$, Liju Zong ${ }^{1 *}$, Jing Wang ${ }^{2}$, Xirun Wan ${ }^{1}$, Fengzhi Feng ${ }^{1}$, Yang Xiang ${ }^{1 凶}$ \\ 1. Department of Obstetrics and Gynecology, Peking Union Medical College Hospital, Chinese Academy of Medical Sciences, Beijing 100730, PR China. \\ 2. Department of Pathology, Peking Union Medical College Hospital, Chinese Academy of Medical Sciences, Beijing 100730, PR China. \\ *These authors contributed equally to this work. \\ $\triangle$ Corresponding author: Yang Xiang, Department of Obstetrics and Gynecology, Peking Union Medical College Hospital, Chinese Academy of Medical \\ Sciences, Beijing 100730, PR China. Tel.: +86 106529 6068; Fax: +86 106512 4875; E-mail: xiangy@pumch.cn \\ (c) Ivyspring International Publisher. This is an open access article distributed under the terms of the Creative Commons Attribution (CC BY-NC) license \\ (https://creativecommons.org/licenses/by-nc/4.0/). See http://ivyspring.com/terms for full terms and conditions.
}

Received: 2018.06.26; Accepted: 2018.10.03; Published: 2019.01.01

\begin{abstract}
Background: Epithelioid trophoblastic tumors (ETTs) are the rarest type of gestational trophoblastic neoplasias. We investigated the clinical features, treatments, outcomes, and prognostic factors in patients with ETT, and explored potential therapeutic targets.

Methods: We retrospectively analyzed the clinical features, treatments, survival, and prognostic factors of 21 ETT patients treated at our institution between January 2002 and December 2017. Expression levels of programmed cell death 1 (PD-1), PD-1 ligands (PD-Lland PD-L2), B7 family ligands (B7-H3, $\mathrm{B} 7-\mathrm{H} 4$, V-domain Ig suppressor of T cell activation [VISTA], and B7-H6), and CD105 expression were assessed by immunohistochemistry.

Results: Fourteen patients with ETT (66.7\%) presented with irregular vaginal bleeding. Three stage I patients (14.3\%) with normal $\beta$-human chorionic gonadotropin ( $\beta$ - hCG) levels underwent hysterectomy alone. Of the remaining 18 patients who had elevated $\beta$-hCG levels $(85.7 \%), 1$ received chemotherapy and 17 underwent surgery and multi-agent chemotherapy. After treatment, 17 patients $(81.0 \%)$ achieved complete remission (2 of whom [11.8\%] later relapsed) and 4 (19.0\%) with stage IV died of their disease. On univariate and multivariate analyses, stage IV disease was an independent prognostic factor for overall and disease-free survival $(P<0.001)$. PD-L1, B7-H3, and CD105 were detected in 100\% of samples, PD-L2 and VISTA in $82 \%, \mathrm{~B} 7-\mathrm{H} 6$ in $18 \%$, and B7-H4 was undetectable in ETT cells.

Conclusions: Hysterectomy and metastatic lesion resection are essential for controlling ETT. Surgery plus chemotherapy are recommended for patients with abnormal $\beta$-hCG levels and metastatic disease. PD-L1, PD-L2, B7-H3, VISTA and CD105 are potential therapeutic targets for metastatic ETT.
\end{abstract}

Key words: epithelioid trophoblastic tumor, prognosis, surgery, immune checkpoint

\section{Introduction}

Epithelioid trophoblastic tumor (ETT) is an extremely rare subtype of gestational trophoblastic neoplasia (GTN), accounting for only $1.0-2.0 \%$ of all GTN cases [1, 2]. Initially described as "atypical choriocarcinoma", Shih and Kurman first reported the clinicopathological features of ETT in 1998[3], and the World Health Organization [4] classified ETT as a form of GTN in 2003.

In contrast to choriocarcinoma, ETT arises from chorionic-type intermediate trophoblastic cells and has distinct clinical characteristics [5-7]. Approxima- tely 130 cases of ETT have been reported as of May 2018 , primarily in small retrospective case series $[1$, 7-18]. Because the biologic behaviours of ETT have been reported to be similar to those of placental site trophoblastic tumor (PSTT), and limited evidence is available for the optimal management of ETT, treatment approaches have been derived from case series and PSTT. Surgery is the primary treatment recommended for ETT with nonmetastatic disease. However, patients with ETT might develop metastatic diseases, and some patients will have terminal disease, 
with a mortality rate of $10.0-24.0 \%[3,5,19]$. Chemotherapeutic intervention is usually considered only if surgical intervention is inappropriate or has failed [20]. ETT is generally more refractory to conventional chemotherapy. Therefore, new therapeutic targets are needed to salvage the occasional non-operable patients with metastatic chemoresistant diseases.

Antiangiogenic therapy and immunotherapy targeting immune checkpoints have received significant attention in cancer research. Targeting immune checkpoints such as programmed cell death 1 (PD-1) and its ligand PD-L1 has achieved notable benefit in a variety of cancers. Moreover, a clinical trial of the monoclonal antibody TRC105, which targets the pro-angiogenic modulator CD105, is ongoing [21]. The newly discovered B7 family ligands, B7-H3, B7-H4, V-domain Ig suppressor of $\mathrm{T}$ cell activation (VISTA), and B7-H6, have also been identified as negative immune checkpoint regulators and are targets for cancer immunotherapy [22]. However, the expression patterns of these B7 family ligands and CD105 in ETT remains unknown.

To better understand ETT and identify potential therapeutic targets, we identified a series of patients with ETT who were treated at our institution and investigated their clinical characteristics, management, outcomes, prognostic factors, and expression of immune checkpoint proteins and CD105.

\section{Materials and Methods}

\section{Patients and samples}

Between January 2002 and December 2017, a total of 2129 patients with GTN were admitted to Peking Union Medical College Hospital (Beijing, China) for treatment, 21 (1.0\%) were diagnosed with ETT. All patients, who were identified via a review of institutional databases, were pathologically confirmed to have ETT by at least 2 gynecological pathologists. The study was approved by the Institutional Review Board of Peking Union Medical College Hospital. Medical records were reviewed, and demographic, pathology, treatment, and follow-up data were extracted.

Formalin-fixed, paraffin-embedded tissue specimens from 11 patients were subjected to immunohistochemistry; 6 patients had missing slides or experienced complete tumor necrosis after uterine curettage or chemotherapy, and 4 had undergone surgery at other hospitals before referral to our institution.

\section{Immunohistochemistry and interpretation}

Four-micrometer unstained slides were prepared from representative whole section formalin-fixed paraffin-embedded tumor blocks. All the slides were stained using an automatic immunohistochemistry staining instrument (Leica Biosystems, Buffalo Grove, IL, USA) according to the manufacturer's instructions. The details of primary antibodies and conditions used are available as Supplementary Table S1.

The expression of PD-L1, PD-L2, B7-H3, B7-H4, VISTA, B7-H6 and CD105 in tumor cells were classified as negative, weak, and intense staining. Tumor cells were designated positive, when $\geq 5 \%$ of the tumor cells presented weak or intense staining. The percentages of tumor infiltrating lymphocytes (TILs) that were positive for the aforementioned proteins were estimated, and those with at least 5\% staining were considered positive.

\section{Statistical analyses}

Survival estimates were calculated using the Kaplan-Meier method and compared using the log-rank test. Multivariate analysis was performed using the Cox proportional hazards regression model. All statistical analyses were conducted using the Statistical Package for the Social Sciences for Windows (software version 20.0; IBM Corp., Armonk, NY, USA). A two-sided $P$-value of $<0.05$ was considered statistically significant.

\section{Results}

\section{Clinical characteristics}

The average age at presentation was 35 (range, 24-55) years. The antecedent pregnancies prior to diagnosis were full-term in 16 patients $(76.2 \%)$ whereas $5(23.8 \%)$ underwent an abortion. The median interval between the antecedent pregnancy and diagnosis was 32 (range, 2-264) months. The most common presenting symptom was irregular vaginal bleeding. Pre-treatment serum human chorionic gonadotropin ( $\beta$-hCG) levels ranged from 0.5 to 5 $663.0 \mathrm{IU} / \mathrm{L}$; levels in 5 patients $(23.8 \%)$ were within the normal range $(<5 \mathrm{IU} / \mathrm{L})$. According to the 2000 FIGO staging system for GTN, 4 had FIGO stage IV and they had metastasis to the liver, brain, kidney, spleen, intestinal tract, pelvic lymph nodes, appendix, and omentum. The details of the patients' clinical characteristics are shown in Table 1.

Six patients $(28.6 \%)$ had a history of treatment at other hospitals prior to referral to our facility; 2 had failed multidrug chemotherapy, 3 relapsed after hysterectomy with or without chemotherapy, and 1 with an isolated ETT of the vagina relapsed after resection of a vaginal wall tumor. The details of the patients' initial treatments and outcomes are shown in Table 2.

\section{Treatments algorithm and outcomes}

Three patients (14.3\%) with FIGO stage I disease 
who had non-elevated $\beta$-hCG levels were treated with hysterectomy alone (i.e., without chemotherapy). The remaining 18 patients $(85.7 \%)$ were treated with 1-13 cycles of multi-agent chemotherapy including FAEV (5-fluorouracil, actinomycin-D, etoposide, and vincristine), EMA/CO (etoposide, methotrexate, actinomycin-D/cyclophosphamide and vincristine), and EMA/ EP (etoposide, methotrexate, actinomycin-D/etoposide and cisplatin). The details of our FAEV regimen were described previously [23]. Among the 18 patients treated with chemotherapy, one patient with stage IV who relapsed after undergoing hysterectomy and chemotherapy at another hospital underwent chemotherapy and radiotherapy at our facility; the remaining 17 patients underwent surgery, including resection of the isolated tumor or hysterectomy. An example of pulmonary metastases is shown in Figure 1. The details of their treatments are shown in Table 2.

Seventeen patients $(81.0 \%)$ achieved complete remission (CR) after treatment. Two patients $(9.5 \%)$ had progressive disease and died 3 and 6 months after diagnosis, respectively. Two patients $(9.5 \%)$ achieved partial remission, but refused further treatment and died of multiple organ failure after 34 and 36 months, respectively. Two of the 17 patients who achieved CR over a mean follow-up period of 48 (range, 3-191) months $(11.8 \%)$ experienced a relapse; one relapsed at 3 months of follow-up but eventually achieved CR after chemotherapy and lung lesion resection, while the other relapsed 1 month after treatment and was still undergoing treatment at the time of this writing (Table 2).

In total, 4 patients died during the initial treatment or after relapse, yielding an overall mortality rate of $19.0 \%$. The 5-year overall survival (OS) and disease-free survival (DFS) rates were $72.0 \%(95.0 \%$ confidence interval: $48.0-96.0 \%)$ and $71.0 \%(95.0 \%$ confidence interval: 51.0-90.0\%), respectively (Fig. 2A, B).

\section{Prognostic factors}

Univariate analysis showed stage IV disease as the only risk factor for poor OS. An interval between the antecedent pregnancy and diagnosis of $>120$ months, stage IV disease, metastatic disease, and $\beta$-hCG levels >1 $000 \mathrm{IU} / \mathrm{L}$ were significantly associated with poor DFS. In the multivariate analysis, stage IV disease (hazard ratio: 16.6, 95.0\% confidence interval: 2.9-95.0; $P=0.001$ ) was the only risk factor associated with poor DFS (Table 3). The survival of patients with stage I-III was better significantly than those with stage IV (Fig.2C, D).

Table 1. Clinical characteristics of 21 patients with ETT

\begin{tabular}{llll}
\hline Characteristics & & Number & $\%$ \\
\hline Age (years) & $<40$ & 16 & 76.2 \\
Symptoms & $\geq 40$ & 5 & 23.8 \\
& Vaginal bleeding & 14 & 66.7 \\
& aOthers & 6 & 28.6 \\
AP outcome & Asymptomatic & 1 & 4.8 \\
FIGO stage & Abortion & 5 & 23.8 \\
& Full-term & 16 & 76.2 \\
& I & 8 & 38.1 \\
Interval to AP (months) & II & 3 & 14.3 \\
& III & 6 & 28.6 \\
IV-hCG level (IU/L) & $12-36$ & 4 & 19 \\
& $36-60$ & 5 & 23.8 \\
& $\geq 60$ & 9 & 42.9 \\
Treatment history & $<5$ & 0 & 0 \\
& $5-1000$ & 7 & 33.3 \\
& $\geq 1000$ & 5 & 23.8 \\
& No & 12 & 57.1 \\
& Yes & 4 & 19 \\
\hline
\end{tabular}

ancluding abdominal pain, postpartum fever, hemoptysis, palpable vaginal mass, and hypomenorrhea.

Abbreviations: AP, antecedent pregnancy; ETT, epithelioid trophoblastic tumor; FIGO, International Federation of Gynecology and Obstetrics; $\beta$-hCG, $\beta$-human chorionic gonadotropin

Table 2. Treatments and outcomes of 21 patients with ETT

\begin{tabular}{|c|c|c|c|}
\hline Patients & Treatment at other hospitals and outcome & Treatment at PUMCH and outcome & \\
\hline \multicolumn{4}{|l|}{ Patients referred to PUMCH $(\mathrm{N}=6)$} \\
\hline Stage I $(\mathrm{N}=1)$ & CTx (PR) & LH and CTx & $\mathrm{CR}$ \\
\hline stage II $(\mathrm{N}=1)$ & Tumor resection (relapse) & Metastasectomy and CTx & $\mathrm{CR}$ \\
\hline stage II $(\mathrm{N}=1)$ & TAH (relapse) & Metastasectomy and CTx & $\mathrm{CR}$ \\
\hline stage III $(\mathrm{N}=1)$ & TAH and CTx (relapse) & Metastasectomy and CTx & $\mathrm{CR}$ \\
\hline Stage IV $(\mathrm{N}=1)$ & TAH and CTx (relapse) & Radiotherapy and CTx & DOD \\
\hline Stage IV $(\mathrm{N}=1)$ & CTx (PR) & TAH and CTx & DOD \\
\hline \multicolumn{4}{|l|}{ Patients with initial treatment at PUMCH $(\mathrm{N}=15)$} \\
\hline Stage I with normal $\beta$-hCG $(\mathrm{N}=3)$ & & LH without CTx $(\mathrm{N}=3)$ & $\mathrm{CR}$ \\
\hline \multirow[t]{3}{*}{ Stage I with abnormal $\beta$-hCG and stage II- III $(\mathrm{N}=10)$} & & Tumor resection and CTx $(\mathrm{N}=3)$ & CR \\
\hline & & $\mathrm{LH} / \mathrm{TAH}$ and CTx $(\mathrm{N}=7)$ & $\mathrm{CR}(\mathrm{N}=5)$ \\
\hline & & & Relapse $(\mathrm{N}=2)$ \\
\hline Stage IV ( $\mathrm{N}=2)$ & & TAH and CTx $(\mathrm{N}=2)$ & DOD \\
\hline
\end{tabular}



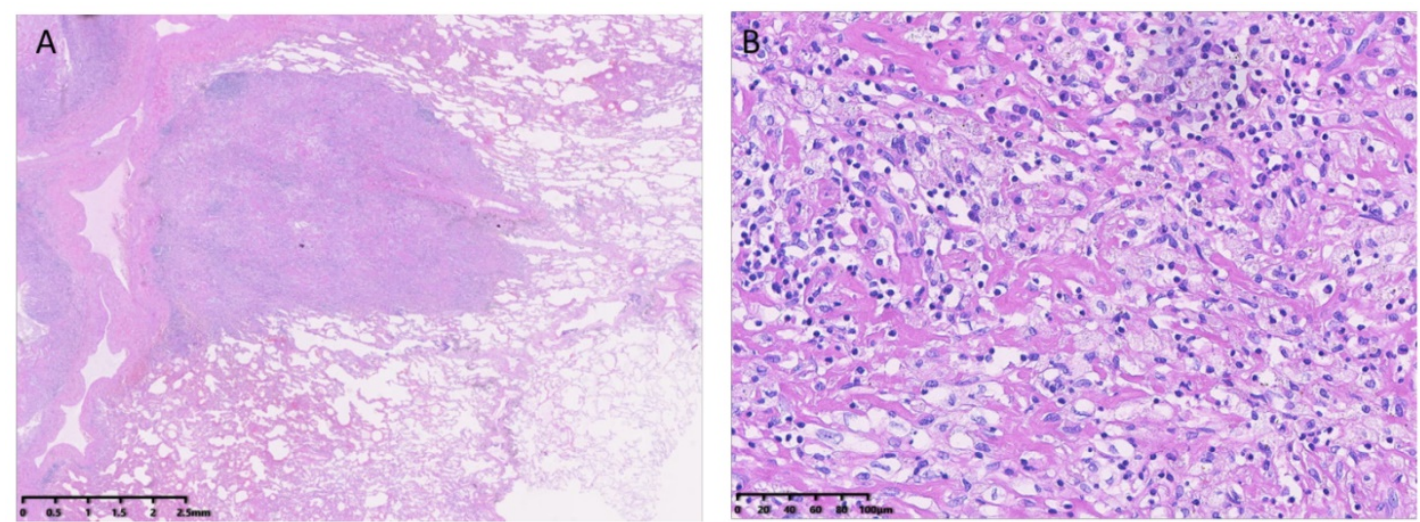

Figure 1. Epithelioid trophoblastic tumor lung metastases. Hematoxylin and eosin stain of a lung metastasis at $(A) \times 10$ and $(B) \times 200$ magnification.
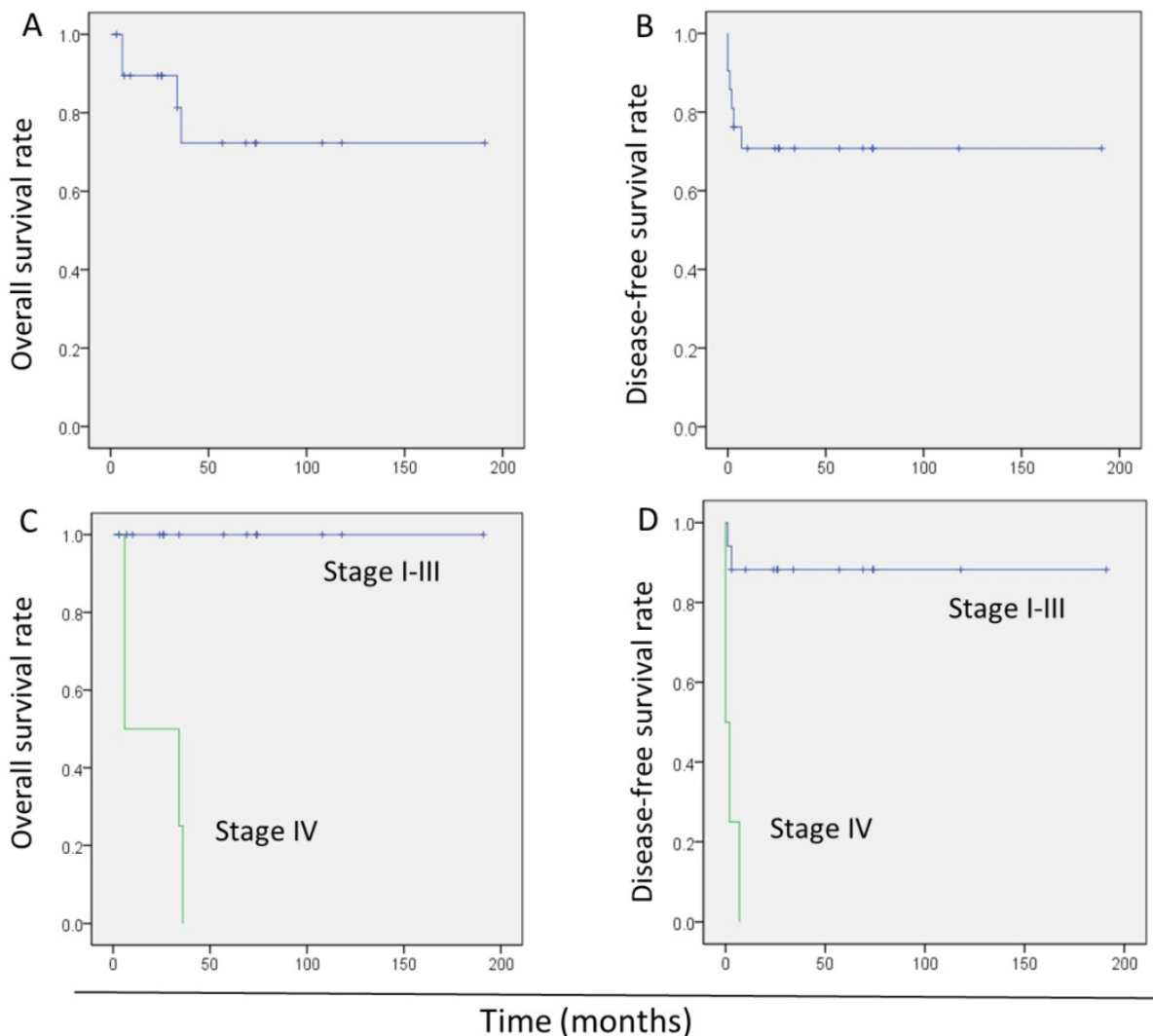

Figure 2. Kaplan-Meier survival curves in 21 patients with epithelioid trophoblastic tumor. (A) Overall survival of all patients. (B) Disease-free survival of all patients. (C) Overall survival of patients with stage I-III disease versus those with stage IV. (D) Disease-free survival of patients with stage I-III and patients with stage IV.

\section{Expression of immune checkpoint regulators and CD105}

The pattern of PD-1, PD-L1, PD-L2, B7-H3, VISTA, and CD105 staining in tumor cell and TILs was predominantly membranous (Fig. 3,4 ), while the localization of B7-H6 was membranous and cytoplasmic in tumor cells and TILs (Fig. 4G). The expression of B7-H4 was not detectable in ETT cells, but it was detected in the positive control (cervical cancer) (Fig. 4I).

In ETT cells, PD-L1, B7-H3, and CD105 were detected in $100 \%$ of ETT cases, PD-L2 and VISTA were detected in $82 \%$, B7-H6 was detected in $18 \%$, and B7-H4 was undetectable (Table 4). In TILs, PD-L1 and VISTA were positive in $100 \%$ of ETT cases, PD- 1 and B7-H3 were positive in $64 \%$, PD-L2 was positive in $91 \%$, B7-H6 was positive in $82 \%$, and B7-H4 was not detectable TILs (Table 4).

Although there was no association between the expressions of immune checkpoints in tumor cells, their expressions had a consistent trend in the same patient.Fig. 5 shows immune checkpoints and CD105 staining in the same fields. 

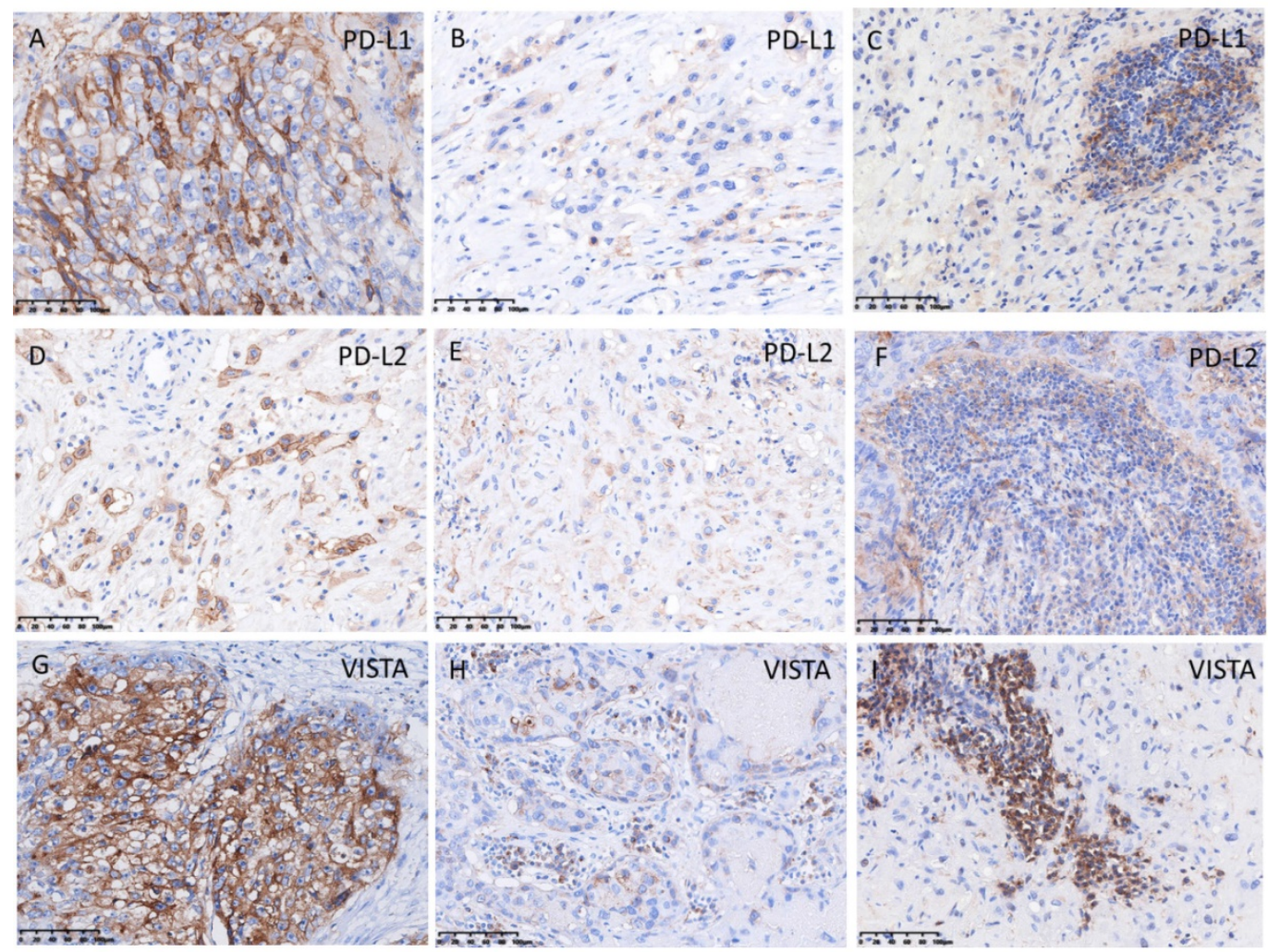

Figure 3. Expression of programmed cell death protein 1 ligands PD-L1, PD-L2, and VISTA in ETT cells and tumor infiltrating lymphocytes (TILs). (A) Intense expression of PD-L1 in ETT cells; (B) weak expression of PD-LI in ETT cells; (C) expression of PD-L1 in TILs; (D) intense expression of PD-L2 in ETT cells; (E) weak expression of PD-L2 in ETT cells; (F) expression of PD-L2 in TILs; (G) intense expression of VISTA in ETT cells; (H) weak expression of VISTA in ETT cells; (I) expression of VISTA in TILs.

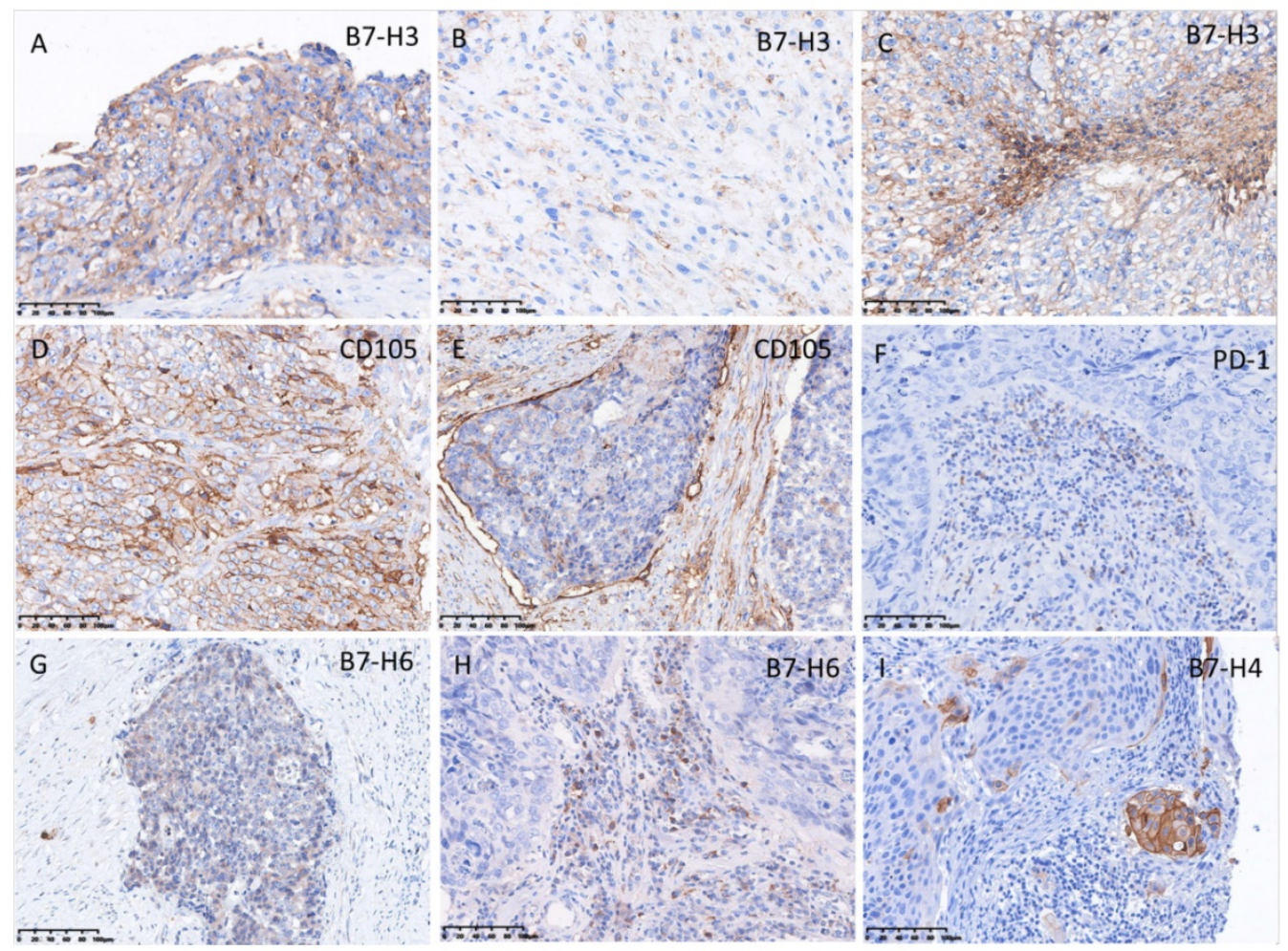

Figure 4. Expression of programmed cell death protein 1 (PD-1), B7-H3, B7-H4, B7-H6, and CD105 in ETT cells and/or tumor infiltrating lymphocytes (TILs). (A) Intense expression of B7-H3 in ETT cells; (B) weak expression of B7-H3 in ETT cells; (C) expression of B7-H3 in TILs; (D) intense expression of CD105 in ETT cells; (E) weak expression of CDI05 in ETT cells; (F) expression of PD-1 in TILs; (G) weak expression of B7-H6 in ETT cells; (H) expression of B7-H6 in TILs; (I) expression of B7-H4 in positive control (cervical cancer). 

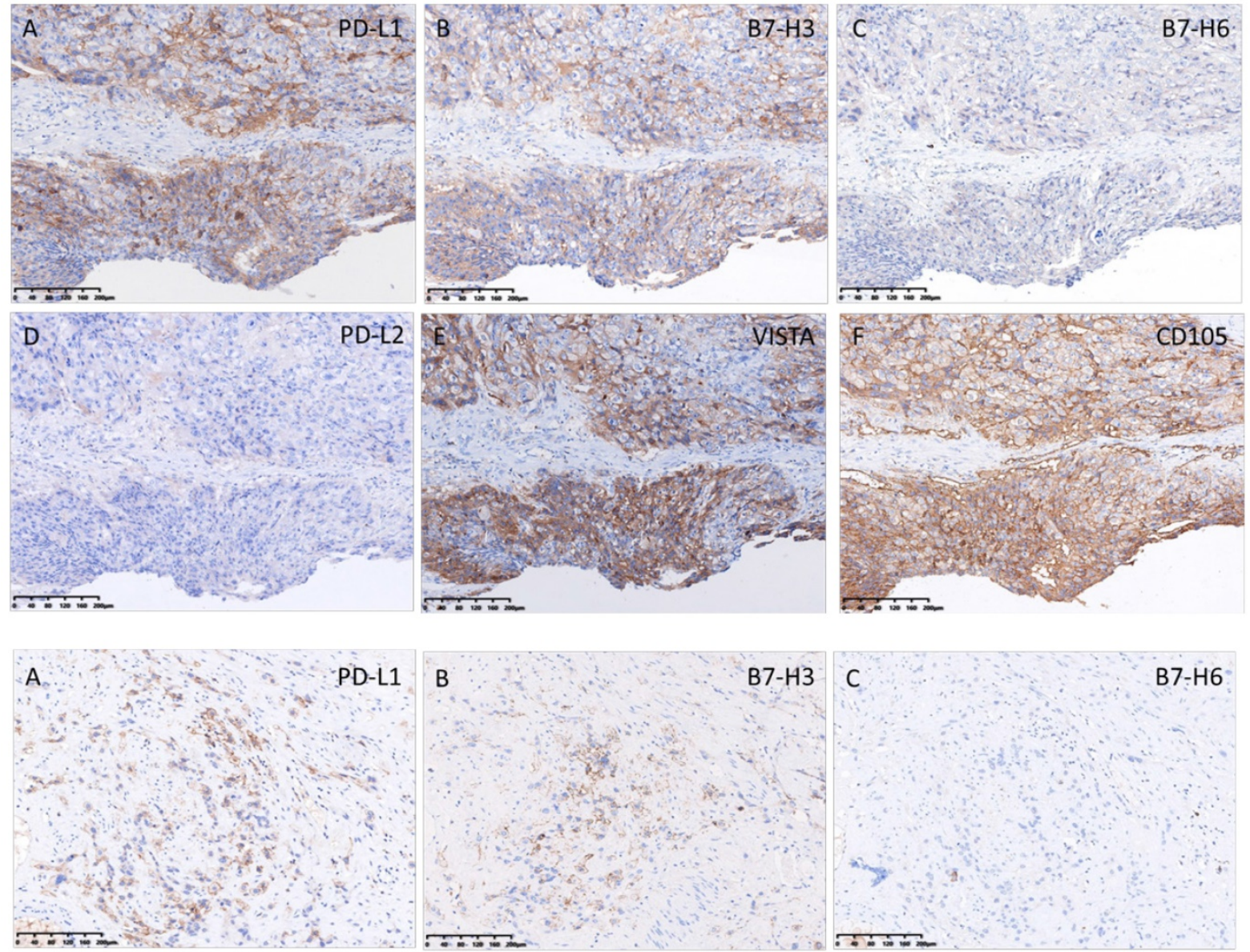

B7- $\mathrm{H} 6$
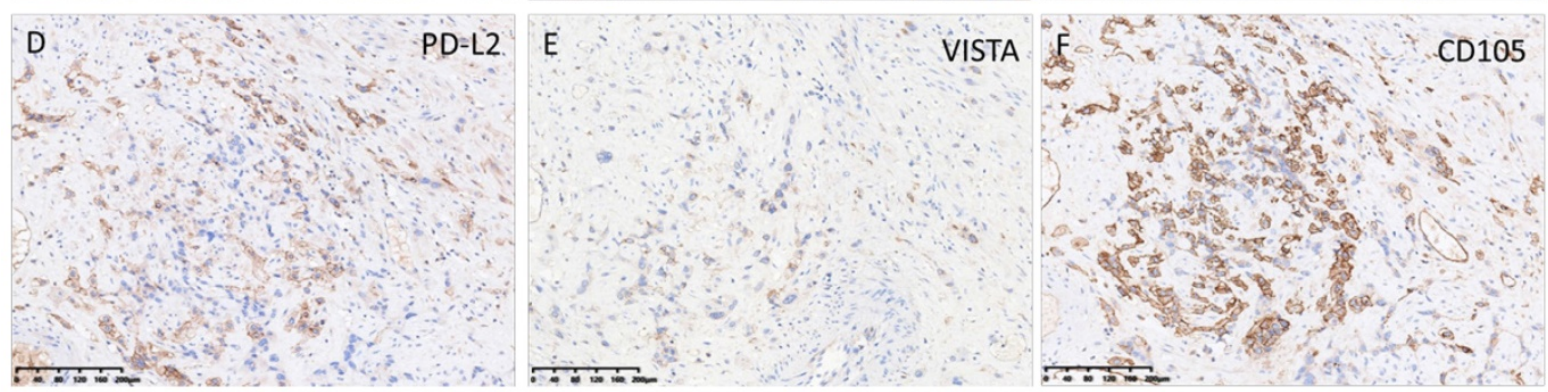

Figure 5. Expressions of immune checkpoint proteins and CD105 in the same fields. Upper panel: (A) intense expression of PD-L1; (B) intense expression of B7-H3; (C) weak expression of B7-H6; (D) negative expression of PD-L2. (E) intense expression of VISTA; (F) intense expression of CD105. Lower panel: (A) weak expression of PD-L1; (B) weak expression of B7-H3; (C) negative expression of B7-H6; (D) weak expression of PD-L2; (E) weak expression of VISTA and (F) intense expression of CD105.

\section{Discussion}

ETT is a rare type of GTN, usually found in women of reproductive age. The incidence of ETT in the present study was $1.0 \%$ among all type of GTN, which is comparable to rates reported in the literature $(1.0-2.0 \%)[1,2]$. Our current study highlights the successful treatment of 21 ETT patients and confirms the necessity of surgery and chemotherapy in the management of this disease. To our knowledge, ours is the largest retrospective case series at a single institution to date. Notably, we also identified PD-L1, PD-L2, B7-H3, VISTA, and CD105 as potential therapeutic targets for metastatic or recurrent ETT.
Diagnosis of ETT remains a challenge owing to its rarity and diverse presentations, which can lead to potential mismanagement and delay in treatment. In line with previous studies [5-7], irregular vaginal bleeding was the most common symptom of ETT in our cohort. ETT can develop after any antecedent pregnancy event, in the present series, $76.2 \%$ of such events were full-term pregnancies, comparable to a mean of $67.0 \%$ in the literature $[3,24,25]$. In previous studies, $14.0 \%$ of patients with ETT had normal levels of $\beta$-hCG while $69.0 \%$ had $\beta$-hCG levels $<2,500 \mathrm{IU} / \mathrm{L}$ $[5,6,25]$. In the present series, 5 patients $(23.8 \%)$ had normal $\beta$-hCG levels and 3 patients $(14.3 \%)$ had $\beta$-hCG levels $>2,500 \mathrm{IU} / \mathrm{L}$. 
Table 3. Univariate and multivariate analysis of 5-year survival rate in 21 patients with ETT

\begin{tabular}{|c|c|c|c|c|c|c|}
\hline \multirow[b]{2}{*}{ Factor } & \multirow[b]{2}{*}{$N$} & \multicolumn{2}{|c|}{$\begin{array}{l}\text { Univariate } \\
\text { analysis }\end{array}$} & \multicolumn{2}{|c|}{$\begin{array}{l}\text { Univariate } \\
\text { analysis }\end{array}$} & \multirow{2}{*}{$\begin{array}{l}\begin{array}{l}\text { Multivariate } \\
\text { analysis }\end{array} \\
P\end{array}$} \\
\hline & & OS rate & $P$ & DFS rate & $P$ & \\
\hline Age (years) & & & 0.312 & & 0.104 & \\
\hline$<40$ & 16 & 86 & & 81 & & \\
\hline$\geq 40$ & 5 & 38 & & 40 & & \\
\hline AP outcome & & & 0.805 & & 0.621 & \\
\hline Abortion & 5 & 75 & & 60 & & \\
\hline Full-term & 16 & 71 & & 75 & & \\
\hline Metastasis & & & 0.129 & & 0.03 & 0.159 \\
\hline No & 8 & 100 & & 100 & & \\
\hline Yes & 13 & 60 & & 53 & & \\
\hline FIGO stage & & & $<0.001$ & & $<0.001$ & 0.001 \\
\hline I-III & 17 & 100 & & 88 & & \\
\hline IV & 4 & 0 & & 0 & & \\
\hline Interval to AP & & & 0.19 & & $<0.001$ & 0.396 \\
\hline$\leq 120 \mathrm{~m}$ & 17 & 87 & & 88 & & \\
\hline$>120 \mathrm{~m}$ & 4 & 33 & & 0 & & \\
\hline Interval to AP & & & 0.618 & & 0.071 & \\
\hline$\leq 60 \mathrm{~m}$ & 14 & 83 & & 86 & & \\
\hline$>60 \mathrm{~m}$ & 7 & 53 & & 43 & & \\
\hline Prior treatment & & & 0.469 & & 0.909 & \\
\hline No & 15 & 85 & & 73 & & \\
\hline Yes & 6 & 50 & & 67 & & \\
\hline$\beta$-hCG level (IU & $\mathrm{U} / \mathrm{L})$ & & 0.256 & & 0.014 & 0.19 \\
\hline$<1000$ & 17 & 82 & & 81 & & \\
\hline$\geq 1000$ & 4 & 50 & & 25 & & \\
\hline
\end{tabular}

Table 4. Expression of immune checkpoint regulators and CD105 in 11 patients with ETT

\begin{tabular}{llllllllll}
\hline & $\begin{array}{l}\text { Immuno- } \\
\text { reactivity }\end{array}$ & PD-1 & $\begin{array}{l}\text { PD- } \\
\text { L1 }\end{array}$ & $\begin{array}{l}\text { PD- } \\
\text { L2 }\end{array}$ & $\begin{array}{l}\text { B7- } \\
\text { H3 }\end{array}$ & $\begin{array}{l}\text { B7- } \\
\text { H4 }\end{array}$ & VISTA B7- & CD \\
\hline Tumor & Negative & $/$ & 0 & 2 & 0 & 11 & 2 & 9 & 0 \\
cells & Weak & $/$ & 1 & 4 & 3 & 0 & 2 & 2 & 2 \\
& Intense & $/$ & 10 & 5 & 8 & 0 & 7 & 0 & 9 \\
\multirow{4}{*}{ TILs } & Negative & 4 & 0 & 1 & 4 & 11 & 0 & 2 & $/$ \\
& Positive & 7 & 11 & 10 & 7 & 0 & 11 & 9 & $/$ \\
\hline
\end{tabular}

Abbreviations: TILs, Tumor-infiltrating lymphocytes; PD-1, programmed death-1 (PD-1); PD-L1, PD-1 ligand 1; PD-L2, PD-1 ligand 2; VISTA, V-domain Ig suppressor of $\mathrm{T}$ cell activation

Approximately $30.0 \%$ of patients reportedly presented with metastatic disease at diagnosis [3, 5]. The uterus is the most common primary site of ETT while the lung is the most common extra-uterine site of metastasis, accounting for $19.0 \%$ of cases [19]. Similar to previous studies reporting high rates of metastasis, $47.6 \%$ of patients in the present series presented with metastatic disease at diagnosis. Extra-uterine cases of ETT are rare and patients with such diseases usually present with low levels of $\beta$-hCG, thus obscuring their diagnosis. In the present study, 3 patients presented with isolated extra-uterine ETT (located in the vagina, pelvis, and lung, respectively), 2 of whom have also been reported separately [26, 27].

Surgical resection is the primary treatment modality and important for ETT present with non-metastatic disease $[2,7,28]$. Previous studies [7, 28 ] have shown that patients with disease confined to the uterus may be cured by primary hysterectomy without adjuvant chemotherapy. In the present series, there was no evidence of disease in patients with ETT confined to the uterus who underwent hysterectomy, those with normal $\beta$-hCG levels who did not receive chemotherapy $(\mathrm{n}=3)$, or those with abnormal $\beta$-hCG levels who received chemotherapy $(n=5)$ during the follow-up period. Although, the present series cannot be used to directly support or refute the efficacy of adjuvant chemotherapy in these patients, the role of surgery alone in patients with normal serum $\beta$-hCG level is certain. Surgery is also important for eradicating metastatic disease and drug-resistant residual disease $[7,28]$. In the present series, all patients with advanced-stage disease underwent multiple surgical procedures, including hysterectomy and resection of isolated tumors. The majority of patients achieved CR $(81.0 \%)$ after treatment.

Although chemotherapy alone is inadequate for advanced-stage and metastatic or locally advanced disease, it still plays an important role in the management of ETT patients. Moreover, it has been reported that systemic multi-agent chemotherapy is advocated for patients with ETT when surgery has failed or is unfeasible (e.g., for metastatic disease)[5]. While several chemotherapy regimens have been used to treat patients with ETT, the optimal chemotherapy regimen has yet to be determined. The preferred first-line chemotherapy regimens are those containing platinum agents, such as EMA/EP, as well as paclitaxel, cisplatin/paclitaxel, etoposide [18]. In the present series, 18 patients received multi-agent chemotherapy, $14(77.8 \%)$ of whom achieved CR. Our data suggest that patients with stage I-III disease may be cured using multidrug chemotherapy and targeted surgery. Notably, our data suggest for the first time that it is reasonable to consider adjuvant chemotherapy in patients with abnormal $\beta$-hCG levels, especially those presenting with extra-uterine tumors.

Data on the survival of patients with ETT are limited. The mortality rate $(10.0-24.0 \%)$ of patients with ETT is higher than that of post-molar GTN (6.5\%) and choriocarcinoma $(13.4 \%)$ [3, 5, 19, 29]. In the present series, the mortality rate was $19.0 \%$ over a follow-up period of 12-189 months, which is comparable to that reported in the literature. The present series also documented 2 patients who relapsed 1 and 3 months after treatment, yielding a recurrence rate of $11.8 \%$ compared to $30.6 \%$ in the literature [19].

As mentioned above, the prognostic factors for 
ETT are unclear because of the rarity of this disease, and are assumed to be similar to those of PSTT. Moreover, the FIGO anatomical stage is a significant prognostic factor for ETT [19]. Our data suggested that FIGO stage IV disease is an independent prognostic factor for both OS and DFS in patients with ETT. A previous study showed that $>4$-year interval between the antecedent pregnancy and ETT diagnosis was an indicator of poor prognosis [7], however, this was not reflected by our data. In the univariate analysis, an interval from the antecedent pregnancy of $>120$ months, FIGO stage IV disease, metastatic disease, and a $\beta$-hCG level (>1 $000 \mathrm{IU} / \mathrm{L}$ ) negatively affected the DFS, which may be important and deserves more attention.

The treatment of patients with stage IV or metastatic ETT remains a challenge. Ghorani [30] and Huang [31] found that pembrolizumab (a humanized monoclonal antibody against PD-1) was effective in patients with chemoresistant GTN. As such, the utilization of immunotherapy for the treatment of choriocarcinoma appears to be an attractive option for patients with ETT. Veras et al. reported that 8 of 14 patients with ETT had detectable levels of PD-L1 in their tumors [32]. In our study, all 11 patients for whom sections were available were positive for PD-L1 expression, suggesting that PD-1 blockade might a yield clinical benefit. PD-L2 and the B7 family ligands, B7-H3, B7-H4, VISTA and B7-H6, are also potential targets for cancer immunotherapy [22]. In our study, we found that both ETT tumor cells and TILs were positive for PD-L2, B7-H3, and VISTA, suggesting that these proteins could also be potential targets for cancer immunotherapy in patients with metastatic ETT. Additionally, Worley et al. reported that a woman with metastatic and refractory choriocarcinoma experienced a durable remission after receiving therapy with TRC105 and bevacizumab [33]. CD105 was detected in all 11 patients who were tested; therefore, anti-CD105 monoclonal antibody therapy may be feasible for metastatic or chemoresistant ETT.

Our study is not without limitations. First, this was a small patient population and all data was collected retrospectively; however, this study included the largest sample size of ETT to our best knowledge and provides useful information in understanding the clinical presentation and treatment of ETT. In addition, only 11 specimens were available for assessment of immune checkpoint proteins and CD105. Even though our sample size is small, ours is the first study to evaluate the immune landscape and CD105 and to identify potential therapeutic targets for metastatic ETT.

\section{Conclusions}

Despite the rarity of ETT and the fact that our study comprised a relatively small number of patients, our data provide additional insight into possible management strategies for patients with this disease. Surgery is critical for the management of ETT, and combined surgery plus chemotherapy are recommended for patients with abnormal $\beta$-hCG levels and metastatic disease. We found that FIGO stage IV disease was the only predictor of poor OS for patients with ETT and influenced the prognosis of ETT. Patients with stage IV disease, an interval of $>120$ months between the antecedent pregnancy and diagnosis, metastatic disease, and $\beta$-hCG levels $(>1$ $000 \mathrm{IU} / \mathrm{L}$ ) should receive particularly close attention. PD-L1, PD-L2, B7-H3, VISTA and CD105 are frequently expressed in ETTs and may thus be potential therapeutic targets.

\section{Abbreviations}

AP: antecedent pregnancy; $\beta$-hCG: $\beta$-human chorionic gonadotropin; CTx: Chemotherapy; CR: complete remission; DFS: disease-free survival; DOD: died of disease; EMA/CO: etoposide, methotrexate, actinomycin-D/cyclophosphamide and vincristine; EMA/EP: etoposide, methotrexate, actinomycin-D/ etoposide and cisplatin; ETT: epithelioid trophoblastic tumor; FAEV: 5-fluorouracil, actinomycin-D, etoposide, and vincristine; FIGO: International Federation of Gynecology and Obstetrics; GTN: gestational trophoblastic neoplasia; IHC: immunohistochemistry; LH: laparoscopic hysterectomy; NA: not available; TAH: total abdominal hysterectomy; OS: overall survival; PD-1: programmed death-1; PD-L1: programmed death-1 ligand 1; PD-L2: programmed death-1 ligand 2; PR: partial response; PUMCH: Peking Union Medical College Hospital; PSTT: placental site trophoblastic tumor; TILs: tumorinfiltrating lymphocytes; VISTA: V-domain Ig suppressor of $\mathrm{T}$ cell activation.

\section{Supplementary Material}

Supplementary table S1.

http://www.jcancer.org/v10p0011s1.pdf

\section{Acknowledgements}

We wish to thank all of the patients and doctors who have participated in this study. This work was supported by grants from the National Natural Science Foundation of China (No. 81672586) and the Chinese Academy of Medical Sciences Initiative for Innovative Medicine (CAMS-2017-I2M-1-002). 


\section{Author contributions}

$\mathrm{YJ}, \mathrm{WX}, \mathrm{FF}$ and $\mathrm{XY}$ participated in the design of the study, analyzed and interpreted the patient data, performed the statistical analysis. YJ and ZL participated in writing the manuscript. WJ performed the immunohistochemistry. The final version of the manuscript has been reviewed and approved for publication by every author.

\section{Ethics Committee Approval and Patient Consent}

This study was conducted according to the ethical standards contained in the Declaration of Helsinki, and in national and international guidelines. The study was approved by the Institutional Review Board of Peking Union Medical College Hospital (Beijing, China). Patient consent and approval from the Institutional Research Ethics Committee of Peking Union Medical College Hospital were obtained to use of these clinical materials for research purposes.

\section{Competing Interests}

The authors have declared that no competing interest exists.

\section{References}

1. Lybol C, Thomas CM, Bulten J, van Dijck JA, Sweep FC, Massuger LF. Increase in the incidence of gestational trophoblastic disease in The Netherlands. Gynecol Oncol. 2011; 121: 334-8.

2. Li J, Shi Y, Wan X, Qian H, Zhou C, Chen X. Epithelioid trophoblastic tumor: a clinicopathological and immunohistochemical study of seven cases. Med Oncol. 2011; 28: 294-9.

3. Shih IM, Kurman RJ. Epithelioid trophoblastic tumor: a neoplasm distinct from choriocarcinoma and placental site trophoblastic tumor simulating carcinoma. Am J Surg Pathol. 1998; 22: 1393-403.

4. Genest DR BR, Fisher RA. Gestational trophoblastic disease. In: F.A. T, editor. WHO Classification of Tumours Pathology and Genetics of Tumours of the Breast and Female Genital Organs. Lyon, France: IARC Press. 2003;:250-4.

5. Palmer JE, Macdonald M, Wells M, Hancock BW, Tidy JA. Epithelioid trophoblastic tumor: a review of the literature. J Reprod Med. 2008; 53: 465-75.

6. Horowitz NS, Goldstein DP, Berkowitz RS. Placental site trophoblastic tumors and epithelioid trophoblastic tumors: Biology, natural history, and treatment modalities. Gynecol Oncol. 2017; 144: 208-14.

7. Davis MR, Howitt BE, Quade BJ, Crum CP, Horowitz NS, Goldstein DP, et al. Epithelioid trophoblastic tumor: A single institution case series at the New England Trophoblastic Disease Center. Gynecol Oncol. 2015; 137: 456-61.

8. Rodríguez-Trujillo A, Martínez-Serrano MJ, Saco A, Torné A. Two cases of epithelioid trophoblastic tumors in postmenopausal women. Menopause. 2017; 24: 1304-8.

9. Stanculescu RV, Bausic V, Vladescu TC, Vasilescu F, Bratila E. Epithelioid trophoblastic tumor: a case report and literature review. Rom J Morphol Embryol. 2016; 57: 1365-70.

10. Usubutun A, Selcuk I, Boyraz G, Tuncer ZS. An incidentally diagnosed epithelioid trophoblastic tumor in hysterectomy. Pathologica. 2015; 107: 201-4.

11. Kim JH, Lee SK, Hwang SH, Kim JS, Yoon G, Lee YY, et al. Extrauterine epithelioid trophoblastic tumor in hysterectomized woman. Obstet Gynecol Sci. 2017; 60: 124-8.

12. Kageyama S, Kanoto M, Sugai Y, Suto T, Nagase S, Osakabe M, et al. MR imaging of uterine epithelioid trophoblastic tumor: a case report. Magn Reson Med Sci. 2016; 15: 411-5.

13. Ohya A, Higuchi K, Shimojo H, Fujinaga Y, Kadoya M, Shiohara S. Epithelioid trophoblastic tumor of the uterus: A case report with radiologic-pathologic correlation. J Obstet Gynaecol Res. 2017; 43: 1360-5.

14. Park JW, Bae JW. Epithelioid Trophoblastic Tumor in a Postmenopausal Woman: A Case Report. J Menopausal Med. 2016; 22: 50-3.

15. Zhang X, Zhou C, Yu M, Chen X. Coexisting epithelioid trophoblastic tumor and placental site trophoblastic tumor of the uterus following a term pregnancy: report of a case and review of literature. Int J Clin Exp Pathol. 2015; 8: 7254-9.
16. Zhou F, Lin K, Shi H, Qin J, Lu B, Huang L. Atypical postcesarean epithelioid trophoblastic lesion with cyst formation: a case report and literature review. Hum Pathol. 2015; 46: 1036-9.

17. Imamura $\mathrm{Y}$, Tashiro $\mathrm{H}$, Saito $\mathrm{F}$, Takaishi $\mathrm{K}$, Ohba $\mathrm{T}$, Fukunaga $\mathrm{M}$, et al. Choriocarcinoma coexisting with epithelioid trophoblastic tumor of the uterine horn. Gynecol Oncol Rep. 2015; 14: 31-3.

18. Sobecki-Rausch J, Winder A, Maniar KP, Hoekstra AV, Berry E, Novak K, et al. Surgery and Platinum/Etoposide-Based Chemotherapy for the Treatment of Epithelioid Trophoblastic Tumor. Int J Gynecol Cancer. 2018; 28: 1117-22.

19. Zhang X, Lu W, Lu B. Epithelioid trophoblastic tumor: an outcome-based literature review of 78 reported cases. Int J Gynecol Cancer. 2013; 23: 1334-8.

20. Bolze PA, Attia J, Massardier J, Seckl MJ, Massuger L, van Trommel N, et al. Formalised consensus of the European Organisation for Treatment of Trophoblastic Diseases on management of gestational trophoblastic diseases. Eur J Cancer. 2015; 51: 1725-31.

21. Apolo AB, Karzai FH, Trepel JB, Alarcon S, Lee S, Lee MJ, et al. A phase II clinical trial of TRC105 (anti-endoglin antibody) in adults with advanced/metastatic urothelial carcinoma. Clin Genitourin Cancer. 2017; 15: 77-85.

22. Ni L, Dong C. New B7 Family Checkpoints in Human Cancers. Mol Cancer Ther. 2017; 16: 1203-11.

23. Yang J, Xiang Y, Wan X, Feng F, Ren T. Primary treatment of stage IV gestational trophoblastic neoplasia with floxuridine, dactinomycin, etoposide and vincristine (FAEV): A report based on our 10-year clinical experiences. Gynecol Oncol. 2016; 143: 68-72.

24. Scott EM, Smith AL, Desouki MM, Olawaiye AB. Epithelioid trophoblastic tumor: a case report and review of the literature. Case Rep Obstet Gynecol. 2012; 2012: 862472

25. Allison KH, Love JE, Garcia RL. Epithelioid trophoblastic tumor: review of a rare neoplasm of the chorionic-type intermediate trophoblast. Arch Pathol Lab Med. 2006; 130: 1875-7.

26. Jiang F, Xiang Y, Guo LN. Laparoscopic diagnosis and treatment of an isolated epithelioid trophoblastic tumor in recto-uterine pouch. J Obstet Gynaecol Res. 2018; 44: 960-5.

27. Zhao J, Xiang Y, Zhao D, Ren T, Feng F, Wan X. Isolated epithelioid trophoblastic tumor of the vagina: a case report and review of the literature. Onco Targets Ther. 2013; 6: 1523-6.

28. Taylor F HB. Pharmacotherapy of placental site and epithelioid trophoblastic tumours. Expert Opin Orphan Drugs. 2015; 3: 75-8.

29. Agarwal R, Alifrangis C, Everard J, Savage PM, Short D, Tidy J, et al. Management and survival of patients with FIGO high-risk gestational trophoblastic neoplasia: the U.K. experience, 1995-2010. J Reprod Med. 2014; 59: 7-12.

30. Ghorani E, Kaur B, Fisher RA, Short D, Joneborg U, Carlson JW, et al. Pembrolizumab is effective for drug-resistant gestational trophoblastic neoplasia. Lancet. 2017; 390: 2343-5.

31. Huang M, Pinto A, Castillo RP, Slomovitz BM. Complete serologic response to pembrolizumab in a woman with chemoresistant metastatic choriocarcinoma. J Clin Oncol. 2017; 35: 3172-4.

32. Veras E, Kurman RJ, Wang TL, Shih IM. PD-L1 Expression in Human Placentas and Gestational Trophoblastic Diseases. Int J Gynecol Pathol. 2017; 36: $146-53$

33. Worley MJ, Jr., Elias KM, Horowitz NS, Quade BJ, Berkowitz RS. Durable remission for a woman with refractory choriocarcinoma treated with anti-endoglin monoclonal antibody and bevacizumab: A case from the New England Trophoblastic Disease Center, Brigham and Women's Hospital and Dana-Farber Cancer Institute. Gynecol Oncol. 2018; 148: 5-11. 